Commun. math. Phys. 3, 181-186 (1966)

\title{
Inhomogeneous Completion of Non-Compact Semisimple Groups with the Maximal Compact Subgroup as Little Group
}

\author{
Y. NE'EMAN \\ Tel-Aviv University, Ramat Aviv, Israel
}

Received April 25, 1966

\begin{abstract}
An Abelian set of generators $T^{u}$ is adjoined to a semisimple noncompact Lie group, and its metric chosen so as to have a subset of representations with $T^{u} T_{u}>0$. We study the dimensionality and transformation properties of the $T^{u}$ sub-algebra under the homogeneous part, such that they make its maximal compact subgroup into the stability group ("little group") for the above representations. The problem is related to the appearance of inhomogeneous non-compact groups in quantum mechanical problems.
\end{abstract}

\section{Introduction}

Infinite-dimensional unitary representations of non-compact algebras generate the full spectrum of bound solutions of various problems in Quantum Mechanics [1] - [9]. However, in some cases the relevant SGA (spectrum-generating algebra) contains a set of translation-like Abelian generators, in addition to the semi-simple non-compact ones. One thus encounters an "inhomogeneous" SGA, bearing some resemblance to the Poincare algebra. This fact is especially useful whenever that subalgebra of the homogeneous part of the SGA which commutes with a translation-vector (i.e. the generators of the stability subgroup or "little-group") coincides with the maximal compact subalgebra. The "length" of this translation-vector (e.g. of the 4-momentum in the Poincaré case) may be fixed by the particular conditions of the problem. In such cases, the set of bound solutions is no longer given by a unitary infinite-dimensional representation of the discrete type; instead it may consist of just one or several representations (finite of course) of the (compact) little group, just as an irreducible representation of the Poincaré group is given by $M^{2}$ and one spin $j$. The infinite-dimensionality is no longer of the discrete type, and resides entirely in the quasimomenta $t^{u}$.

In this study, we shall answer the following question: what is the representation-structure characterizing the translation-like set of 
generators under the homogeneous ones such that it yield the maximal compact subalgebra as the stability subalgebra? We shall answer this question for the inhomogeneous extensions of semi-simple classical groups.

\section{Generators and commutators}

Denote the set of translation operators by $T^{u}$, the generators of the maximal compact sub-algebra as $K^{i}$, the non-compact generators of the homogeneous semi-simple SGA by $N^{a}$. We have,

$$
\left[K^{i}, K^{j}\right]=i f^{i j}{ }_{k} K^{k}, \quad i, j, k=1 \ldots n_{K}
$$

with $f^{i j}{ }_{k}$ totally antisymmetric in any two indices,

$$
\left[K^{i}, N^{a}\right]=i c^{i a}{ }_{b} N^{b}, \quad i=1 \ldots n_{K}, a, b=1 \ldots n_{N}
$$

where the $N^{b}$ behave like some finite set of representations of the $K^{i}$,

$$
c^{i a_{b}}=C^{i\left(a_{b}\right)}
$$

with $C^{i}$, the $n_{N}$-dimensional representation of the $K^{i}$.

We also have

$$
\left[N^{a}, N^{b}\right]=-i f^{a b}{ }_{j} K^{j}
$$

the $f^{a b}{ }_{j}$ being totally antisymmetric structure-constants of the compact algebra generated by the $K^{i}$ and the $N^{a^{\prime}}$, the latter behaving under commutation according to

$$
N^{a^{\prime}} \sim i N^{a}
$$

though their actual structure may be entirely different. The $\mathrm{N}^{a}$ are Hermitian and finite; the $N^{a}$ are Hermitian and infinite, as far as their quantum-mechanical application goes. In addition,

$$
\left[T^{u}, T^{v}\right]=0 \text {. }
$$

They span a space of $n_{T}$ dimensions; they thus form the basis for a nonHermitian $n_{T^{-}}$dimensional representation $H$ of the $\left\{K^{i} ; N^{a}\right\}$ non-compact algebra, with indefinite metric $g^{u v}$. Alternatively we may describe them as spanning a finite-dimensional Hermitian representation $H^{\prime}$ of $\left\{K^{i} ; N^{a^{\prime}}\right\}$; with $g^{u v}$ replaced by a positive-definite $g^{\prime} u v$,

where

$$
\left[K^{i}, T^{u}\right]=i h^{i u}{ }_{v}^{v} ;\left[N^{a^{\prime}}, T^{u}\right]=i h^{\prime a u}{ }_{w} T^{w}
$$

$$
h^{l m}{ }_{n}=H^{l\left(m_{n)}\right.} \quad l=1 \ldots\left(n_{K}+n_{N}\right) ; m, n=1 \ldots n_{T} .
$$

To ensure that $\left\{K^{i}\right\}$ be in the stability subgroup, we should be able to "rotate" a certain set of quasi-time-like $T^{u}$ so that they will lie entirely in a subspace which is not acted upon by the $K^{i}$. This requires the existence of at least one $K^{i}$-scalar in the reduction of the $H^{l}$ matrices with respect to the $\left\{K^{i}\right\}$ subalgebra. We pick the signature so that this component of $g^{u v}$ has the value +1 for a diagonal metric. We further require 
that $H^{l}$ contain only one $K^{i}$-scalar in this reduction; and we give a value -1 to all other components of this quasi-Minkowskian metric. Our quasi-time-like $T^{u}$ will thus have

$$
\sum_{u, v=1}^{n \mathrm{~T}} g^{u v} T_{u} T_{v}=t^{2}>0
$$

and our aim is to pick out the $H^{l}$ representation so that for

we should have

$$
\left[X^{m}, \mathbf{T}\right]=0, t^{2}>0,\left\{X^{m}\right\} \subset\left\{K^{i}, N^{a}\right\}
$$

$$
\left\{X^{m}\right\} \equiv\left\{K^{i}\right\}
$$

Clearly, $\mathbf{T}$ may be rotated so that it lies entirely in the $g^{u u}=1$ direction, so that $\left\{K^{i}\right\} \subset\left\{X^{m}\right\}$. However, to ensure that the $X^{m}$ do not constitute a subalgebra larger than the $K^{i}$, we have to impose further restrictions on the $H$. This can be done by an explicit check in each particular class of inhomogeneous classical non-compact algebras; in fact, we shall see that it will correspond to taking the smallest representation of $\left\{K^{i}, M^{a}\right\}$ which contains a $K^{i}$-scalar (and only one).

\section{ISL $(\mathbf{n}, \mathbf{C})$}

To pick $H$, we have to go over to the compact $\left\{K^{i}, N^{a^{\prime}}\right\}$. The $\left\{K^{i}\right\}$ is here $S U(n)$, the $\left\{N^{a}\right\}$ behave under them as $\left(\mathbf{n}^{2}-\mathbf{1}\right)$, the basis of the adjoint representation of $U(n)$. Here $n=n_{K}=n_{N}$. We pick out the $n^{2}-1$ semi-simple generators in $\left\{K^{i}\right\}$ and combine them in $2^{-1 / 2}\left(K^{i} \pm i N^{i}\right)$ with the suitable components of $N^{i}$ to generate the compact group $S U(n)^{(+)} \times S U(n)^{(-)}$. We now take either one of the representations (or some linear combination as a direct sum)

$$
\mathscr{D}\left(n, n^{*}\right), \mathscr{D}\left(n^{*}, n\right)
$$

of that group. Under the $S U(n)$ of the $\left\{K^{i}\right\}$, each one splits into $\left(\mathbf{n}^{2}-1\right) \oplus \mathbf{1}$; taking the positive linear combination of the $S U(n)$ scalar we get our single real $\left\{K^{i}\right\}$ scalar. The $n, n^{*}$ being the smallest (defining) representations in $S U(n)^{(+)}$and $S U(n)^{(-)}$respectively, we also have the minimal answer. The quasi-Minkowskian space is then $n^{2}$ dimensional. A detailed discussion of this case was given by RüHL [10]. Note that in that particular case, both spaces were used, i.e. $n_{T}=2 n^{2}$, so as to satisfy a particular requirement of the existence of a parity operation. However, there is one case in which $n^{2}$ replaces $2 n^{2}$ even though parity is defined; this is the Poincaré group $I S L(2, C)$, as $2^{*} \equiv 2$ in $S U(2)$. The parity operation requires $\mathbf{n} \rightarrow-\mathbf{n}^{\prime}$; this generally requires a second irreducible representation, if $n^{*} \neq n$. In space-time or momentum space, the translations form a $\left(\frac{1}{2}, \frac{1}{2}\right)$ basis, reducing to 13 Commun. math. Phys., Vol. 3 
$3+1$ under space rotations. The symmetric combination 3 of $\frac{1}{2} \times \frac{1}{2}$ inverts under reflection, the antisymmetric 1 doesn't.

To convince ourselves that equ. (11) is fulfilled, we note that the time-like $\mathscr{D}\left(n, n^{*}\right)_{1}$ or $\mathscr{D}\left(n^{*}, n\right)_{1}$, being scalars under the summed action of $S U(n)^{(+)}$and $S U(n)^{(-)}$, and not being scalar under both subgroups independently, they can also not be scalars under their subtracted action.

\section{I0 (n, C)}

A similar treatment here would lead us to go over to $O(n, R)^{(+)} \times$ $\times O(n, R)^{(-)}$and take the basis

$$
\mathscr{D}(n, n)
$$

which reduces to $1 \oplus \mathbf{1} / 2 n(n-1) \oplus[1 / 2 n(n+1)-1]$ under the compact $O(n, R)$ subalgebra.

However, for $n<7$, the spinor representation is smaller than $n$; we may thus use for $n=6 \mathscr{D}\left(s, s^{*}\right), \mathscr{D}\left(s^{*}, s\right)$, some direct linear combination, or their direct sum (if we require a parity operation); $s^{*}$ denoting the conjugate type of spinor. When it doesn't exist, i.e. for $n<6$ we use $\mathscr{D}(s, s)$.

\section{ISL (n, R)}

The compact subgroup is $O(n, R) \rightarrow\left\{K^{i}\right\}, n_{K}=\frac{1}{2} n(n-1)$. The non-compact generators behave like an irreducible 2 nd rank symmetric tensor with $n_{N}=\frac{1}{2} n(n+1)-1$. The compact $\left\{K^{i}, N^{a^{\prime}}\right\}$ is $S U(n)$; we are after a finite representation of $S U(n)$ containing an $O(n)$ scalar. This is not the adjoint representation, as the $O(n)$ scalar in the product

$$
\mathbf{n} \times \mathbf{n}^{*}=\left(\mathbf{n}^{2}-\mathbf{1}\right)+\mathbf{1}
$$

is also an $S U(n)$ scalar 1. To get our answer we have to take the first $\mathscr{D}^{*} \times \mathscr{D}$ product with two $O(n)$ scalars. The $\mathscr{D}(2,0, \ldots, 0)$ representation of $S U(n)$ has $\frac{n}{2}(n+1)$ components; under $S O(n)$ it further reduces into a scalar and the traceless symmetric tensor of rank 2 . When multiplied by its conjugate it will of course thus contain two $S O(n)$ scalars, one of which will coincide with the $S U(n)$ tensor, and the other will occur in the representation $\mathscr{D}(2,0, \ldots, 0,2)$, with dimensionality

$$
d=\left[\frac{n}{2}(n+1)\right]^{2}-n^{2}=\frac{n^{2}}{4}\left(n^{2}+2 n-3\right) .
$$

In the chain $S U(n) \rightarrow O(n), O(n)$ is a maximal subgroup, so that our $\mathscr{D}(2,0, \ldots, 0,2)$ is the quasi-Minkowskian fulfilling equ. (11). 
IU $(\mathbf{n}, \mathbf{m})$

The maximal compact subgroup is $U(n) \times U(m)$. The $\left\{K^{i}, N^{a^{\prime}}\right\}$ is $U(n+m)$. Its adjoint representation $\mathscr{D}\left[(n+m)^{2}-1\right]$ answers our quest. It contains a $K^{i}$ scalar, as can be seen in the product of $\mathscr{D}(n+m) \otimes$ $\mathscr{D}(n+m)^{*}$ when taking the $U(n) \times U(m)$ content,

$$
[(\mathbf{n}, \mathbf{1}) \oplus(\mathbf{1}, \mathbf{m})] \times\left[\left(\mathbf{n}^{*}, \mathbf{1}\right) \oplus\left(\mathbf{1}, \mathbf{m}^{*}\right)\right]
$$

$=\left[\left(\mathbf{n}^{2}-\mathbf{1}, \mathbf{1}\right) \oplus\{(\mathbf{1}, \mathbf{1}) \oplus-(\mathbf{1}, \mathbf{1})\} \oplus\left(\mathbf{1}, \mathbf{m}^{2}-\mathbf{1}\right)\right] \oplus[(\mathbf{1}, \mathbf{1}) \oplus(\mathbf{1}, \mathbf{1})]$.

The $U(n) \times U(m)$ algebra is a maximal sub-algebra of $U(n+m)$, so that equ. (11) is fulfilled.

\section{IO $(p, q)$}

The maximal compact subgroup is $O(p, R) \times O(q, R)$. The $\left\{K^{i}, N^{a^{\prime}}\right\}$ is $O(p+q, R)$. Our answer is found in its symmetric traceless representation with $n_{T}=\frac{1}{2}(p+q)(p+q+1)-1$ appearing in the product

$$
\begin{aligned}
(p+q) \times & (p+q)=1 \oplus\left[\frac{1}{2}(p+q)(p+q-1)\right] \oplus \\
& \oplus\left[\frac{1}{2}(p+q)(p+q+1)-1 .\right]
\end{aligned}
$$

In terms of $O(p) \times O(q)$, our representation reduces into

$$
\left\{\left[\frac{1}{2} p(p+1)-1\right], 1\right\} \oplus\left\{1,\left[\frac{1}{2} q(q+1)-1\right]\right\} \oplus\{p, q\} \oplus\{1,1\}
$$

the last component being our scalar.

ISp (2n, R)

The $\left\{K^{i}\right\}$ algebra is $S U(n)$, the $\left\{K^{i}, N^{a^{\prime}}\right\}$ is $S p(2 n)$. In it

$$
\mathscr{D}[2 n] \times \mathscr{D}[2 n]=\mathscr{D}[1] \oplus \mathscr{D}\left[2 n^{2}-n-1\right] \oplus \mathscr{D}[n(2 n+1)]
$$

has the $S U(n)$ content,

$$
\begin{gathered}
\left(\mathbf{n}+\mathbf{n}^{*}\right) \times\left(\mathbf{n}+\mathbf{n}^{*}\right)=[\mathbf{1}] \oplus\left[\left(\mathbf{n}^{2}-1\right) \oplus \frac{\mathbf{n}}{2}(\mathbf{n}-1) \oplus \frac{\mathbf{n}}{2}(\mathbf{n}-1)^{*}\right] \oplus \\
\oplus\left[\left(\mathbf{n}^{2}-\mathbf{1}\right) \oplus \mathbf{1} \oplus \frac{\mathbf{n}}{2}(\mathbf{n}+1) \oplus \frac{\mathbf{n}}{2}(\mathbf{n}+1)^{*}\right] .
\end{gathered}
$$

We observe that $\mathscr{D}[n(2 n+1)]$ contains our required $S U(n)$ scalar. $U(n)$ is maximal in the $S p(2 n)$ reduction, and equ. (11) is fulfilled.

\section{ISp (2n, C)}

The $\left\{K^{i}\right\}$ is $U S p(2 n)$; we do as for $S L(n, C)$ and $O(n, C)$ and use the $\left\{K^{i}, N^{a^{\prime}}\right\}$ in the form $U S p(2 n)^{(+)} \times U S p(2 n)^{(-)}$. The answer is then provided by $\mathscr{D}(2 n, 2 n)$ with dimensionality $4 n^{2}$. 


\section{Physical applications}

Attempts aiming at a relativistic extension of $S U(6)$ have included the application [10] of the inhomogeneous $I S L(6, C)$ and $[11] I S U(6,6)$. The actual physical interpretation of the translation-like set of generators seems to lead to difficulties [12]. However, some other problems [3], [4] seem to contain such generators, with restrictions fixing their $p^{2}$. It is also possible that the finite space of bound solutions which arises in such problems as the square-well potential etc., corresponds to situations in which the inhomogeneous SGA projects out a finite little group. This conjecture shall be further studied.

Acknowledgement. The research reported in this document has been sponsored by the Air Force Office of Scientific Research under Grant AF EOAR 66-39 through the European Office of Aerospace Research (OAR) United States Air Force.

\section{References}

[1] Ne'eman, Y.: Proceedings of the 1965 (Second) Coral Gables Conference on Symmetry at High Energies, p. 250. San Francisco: Freeman Publ. 1965.

[2] Dothan, Y., M. Gell-Mann, and Y. Ne'eman: Physics Letters 17, 148 (1965).

[3] 一, and Y. Ne'eman: Proceedings of the 1965 (Second) Athens (Ohio) Conference on Resonant Particles, p. 17.

[4] Cook, T., C. J. Goeber, and B. Sakita: Phys. Rev. Letters 15, 35 (1965).

[5] Ne'eman, Y.: Proceedings of the 1965 Tokyo International Seminar in Theoretical Physics, New York: W. A. Benjamin Tokyo: Syokabo; 1966.

[6] Barut, A. O.: Phys. Rev. 139, B 1433 (1965).

[7] -, P. Budini, and C. Fronsdal: Proc. Roy. Soc. to be pub.

[8] Gotsman, E., and Y. Ne'eman: J. Math. Phys., to be publ.

[9] Barut, A O.: Proceedings of the 1965 Trieste Seminar, in: High energy physics and elementary particles, I.A.E.A., Vienna (1965), p. 679.

[10] RüHL, W.: Proceedings of the 1965 Trieste Seminar, in :High energy physics and elementary particles, I.A.E.A., p. 435. Vienna, 1965.

[11] Charap, J. M., P. T. Matthews, and R. F. Streater: Proc. Roy. Soc. A 290, 24 (1966).

[12] Coleman, S. Phys. Rev. 138, B 1262 (1965). 